\title{
MODELO PARA DESENVOLVIMENTO OBJETOS DE APRENDIZAGEM: DESENVOLVIMENTO DE CURSO SOBRE SISTEMA DE PUBLICAÇÃO CIENTÍFICA PARA EDITORES
}

\author{
RONNIE FAGUNDES DE BRITO* \\ MILTON SHINTAKU* \\ ANDREA FLEURY**
}

\begin{abstract}
RESUMO
A modalidade à distância tem se transformado numa opção viável para repasse de conhecimentos, diante da evolução tecnológica. Entretanto, o desenvolvimento de cursos on-line ainda é um desafio, na medida em que requer uma variedade de profissionais, com perfis distintos. No sentido de facilitar esse desenvolvimento, o presente estudo descreve um modelo de desenvolvimento de objetos de aprendizagem, com aplicação em um curso voltado à ferramenta deeditoração científica. Trata-se de um estudo aplicado, apresentando como resultado um material instrucional implementado, utilizando técnicas que facilitam sua reprodução, com uma metodologia baseada na criação e utilização de rótulos (tags), comuns na informática atual. Tal abordagem facilita 0 desenvolvimento de objetos para aprendizagem por equipes diversas, de forma portável e iterativa. Com isso, contribui-se com a interdisciplinaridade no desenvolvimento de materiais aplicados em ambientes de ensino a distância.
\end{abstract}

PALAVRAS-CHAVE: objetos educacionais; hipermídia; design instrucional.

\begin{abstract}
The distance learning modality has become a viable option for passing on knowledge, given the technological evolution. However, the development of online courses is still challenging requiring a variety of professionals with different profiles. In order to facilitate this development, the present study describes a learning object development model, applied in a course focused on a scientific publishing tool. It is an applied study, presenting as result an instructional material implemented using techniques that facilitate its reproduction, and also presenting a methodology based on the creation and use of labels, common in computer science. Such an approach facilitates the development of learning objects by diverse teams, in a portable and iterative way. With this, it contributes with the
\end{abstract}

\footnotetext{
* Bacharel em Sistemas de Informação pela Universidade Federal de Santa Catarina (2004), mestre em Engenharia e Gestão do Conhecimento pela Universidade Federal de Santa Catarina (2007) e Doutor em Engenharia e Gestão do Conhecimento pela Universidade Federal de Santa Catarina (2012).

** Tecnologista do Instituto Brasileiro de Informação em Ciência e Tecnologia(IBICT).

*** Assistente de pesquisa do Instituto Brasileiro de Informação em Ciência e Tecnologia(IBICT).
} 
interdisciplinarity in the development of materials applied in environments for distance learning.

KEYWORDS: learning objects; hypermedia; instructional design.

\section{EDUCAÇÃO A DISTÂNCIA E OBJETOS DE APRENDIZAGEM}

O ensino à distância (EADX), há muito tempo, tem sido uma opção válida para possibilitar ações que visam ao aperfeiçoamento das pessoas, na medida em que transcende questões de espaço e tempo. Para tanto, a tecnologia sempre foi grande aliada nesse processo, pois tem amparado as atividades por meio da disponibilização de ferramentas que apoiam a execução das tarefas a serem feitas nos contextos da aprendizagem. Com isso, revela-se uma faceta que une educação e tecnologia, entre tantas outras.

Historicamente, como destaca Pereira e Moraes (2009), em breve histórico sobre educação à distância, pode-se dizer que, de forma moderna, o ensino à distância tem seus primórdios ainda no século XVIII (1728), com um curso de taquigrafia por correspondência, ofertado por Cauleb Phillips, utilizando o correio como intermediário. Assim,tem-se quase três séculos desde 0 nascimento dessa forma de ensino.

A educação à distância no Brasil tem início nos anos 20 do século passado, como ressalta Saraiva (1996), com destaque ao uso de tecnologias mais abrangentes a partir dos anos 70. A autora advoga que, pelas características continentais do país e pelos desafios envoltos na educação, é preciso romper com as formas tradicionais de ensino em que a EAD se torna uma das opções mais viáveis.

A utilização de tecnologia no Brasil em educação à distância pode ser observada pelo uso do rádio e televisão, como relatado por Andrade e de Almeida Lopes (2013), muito com o apoio do governo, juntamente com a iniciativa privada. Revela-se o caráter social do ensino à distância intermediado pelo rádio e televisão, porquanto atende a camadas menos favorecidas da população, com programas gratuitos e abrangentes.

$\mathrm{O}$ advento da Internet afetou, consideravelmente, a EAD, visto que oferta a possibilidade da utilização de objetos multimídia e os Ambientes Virtuais de Ensino e Aprendizagem (AVEAs). A tecnologia ofertada com a internet e a web apresenta novos desafios, com isso, desloca-se a questão de discussão para a produção dos objetos de aprendizagem (OAs), que são aplicados 
nesses cenários e podem assumir inúmeras formas e granularidades, desde um objeto digital (figura, audio, etc), a ser utilizado em uma lição, até um curso inteiro.

Testa (2002) revela que a tecnologia em si não é um fator crítico para EAD, mas apenas mais um fator que inclui questões pedagógicas e comportamentais. Apresenta desafios a serem superados nas questões de implementação de cursos EAD, nos quais envolve, não apenas questões pedagógicas, mas também gerenciais e econômicas.

Assim, o objetivo do presente estudo é apresentar um modelo para criação de objetos de aprendizagem digitais a serem oferecidos em AVEAs, com características de portabilidade e reusabilidade. Um modelo desenvolvido e testado em cursos de formação de editores científicos, no que tange ao uso do software livre Sistema de Editoração Eletrônica de Revistas (SEER)/ Open Journal System (OJS), contribuindo com a discussão sobre construção de objetos educacionais e objetos de aprendizagem.

$O$ estudo se alinha ao que Braga et al. (2012) relatam como um desafio à questão do desenvolvimento de objetos educacionais reutilizáveis de qualidade, no qual é considerado um fator decisivo, em que técnicas de engenharia de softwares apresentam potencial para atender esses critérios. Ressalta a adaptação de métodos e técnicas voltadas ao desenvolvimento de ferramentas informatizados para a criação de objetos de aprendizagem digitais, visto que compartilham a essência de serem digitais.

\section{METODOLOGIA}

O desenvolvimento de material instrucional voltado a cenários on-line demanda a atuação de atores com diferentes competências. A produção de objetos de aprendizagem requer comprometimento e atuação de equipe multidisciplinar compostos por designers instrucionais, professores, designers gráficos e programadores (BRAGA et al., 2012). Revela-se, assim, a demanda diversificada de recursos humanos para o desenvolvimento de material instrucional de qualidade para ambientes virtuais de aprendizagem.

Devido às diferenças terminológicas acerca dos materiais instrucionais digitais existentes, muitas delas derivadas da tradução de "learningobject", o presente estudo adapta o estudo de Silveira etal (2003), de forma a apoiar conceitualmente o estudo (quadro 1), 
QUADRO 1 - Caracterização de objetos de aprendizagem

\begin{tabular}{|c|c|c|c|c|}
\hline Granularidade & Elemento & Exemplificação & Atomicidade & Reuso \\
\hline 1 (muito alta) & $\begin{array}{l}\text { Elementos } \\
\text { educacionais }\end{array}$ & $\begin{array}{l}\text { Mídias brutas: } \\
\text { Imagens, sons, } \\
\text { vídeos, textos }\end{array}$ & Atômico & $\begin{array}{c}\text { São bases para } \\
\text { reuso }\end{array}$ \\
\hline 2 (alta) & $\begin{array}{ll}\text { Objeto } & \text { de } \\
\text { Informação }\end{array}$ & $\begin{array}{c}\text { Webpages, } \\
\text { exercícios, tarefas, } \\
\text { uma peça única que } \\
\text { possui foco }\end{array}$ & $\begin{array}{l}\text { Composto de } \\
\text { recursos } \\
\text { educacionais }\end{array}$ & $\begin{array}{c}\text { Reutilizados } \\
\text { como unidades } \\
\text { independentes } \\
\text { ou reutilizando } \\
\text { partes do objeto }\end{array}$ \\
\hline 3 (média) & Objeto educacional & $\begin{array}{c}\text { Possui um objetivo } \\
\text { educacional }\end{array}$ & $\begin{array}{c}\text { São compostos } \\
\text { de objetos de } \\
\text { informação e } \\
\text { recursos } \\
\text { educacionais }\end{array}$ & $\begin{array}{c}\text { Reutilizados } \\
\text { como unidades } \\
\text { independentes } \\
\text { ou podem ser } \\
\text { decompostos e } \\
\text { reutilizadas } \\
\text { partes }\end{array}$ \\
\hline 4 (baixa) & $\begin{array}{ll}\text { Objeto } & \text { de } \\
\text { aprendizagem }\end{array}$ & $\begin{array}{l}\text { Cursos compostos de } \\
\text { objetos educacionais }\end{array}$ & $\begin{array}{l}\text { Compostos de } \\
\text { objetos } \\
\text { educacionais }\end{array}$ & $\begin{array}{l}\text { Reutilizados em } \\
\text { sua totalidade }\end{array}$ \\
\hline 5 (muito baixa) & $\begin{array}{l}\text { Ambiente } \\
\text { aprendizado }\end{array}$ & $\begin{array}{c}\text { Combinação de } \\
\text { conteúdos e } \\
\text { tecnologia para } \\
\text { interações de ensino }\end{array}$ & \begin{tabular}{|c|} 
Compostos de \\
componentes de \\
aprendizado
\end{tabular} & $\begin{array}{l}\text { O ambiente não } \\
\text { é reutilizado } \\
\text { com facilidade, } \\
\text { mas sim seus } \\
\text { recursos }\end{array}$ \\
\hline
\end{tabular}

No que concerne à avaliação do objeto de aprendizagem, utilizaram-se os critérios levantados por Braga et al (2012), baseadas em: a) normas de qualidade de software ISO/IEC 9126; b) itens de avaliação sugeridos pela Learning ObjectReviewInstrument (LORI); e c) índices de satisfação sugeridos pela Computer Education Management Association (CEdMA, 2001, apresentadas no quadro 2.

QUADRO 2 - Critérios para avaliação de OAs

\begin{tabular}{|l|l|l|}
\hline Característica & descrição & norma \\
\hline $\begin{array}{l}\text { Habilidades } \\
\text { Didático }\end{array}$ & $\begin{array}{l}\text { O OA deve ser capaz de mostrar ao aluno o o } \\
\text { objetivo do aprendizado a que se propõe. Sendo }\end{array}$ & LORI \\
\hline
\end{tabular}




\begin{tabular}{|l|l|l|}
\hline Pedagógicas & $\begin{array}{l}\text { esse objetivo alinhado às metas de } \\
\text { aprendizagem e características dos alunos. É } \\
\text { desejável que O OA forneça feedback suficiente } \\
\text { para facilitar o aprendizado do aluno. }\end{array}$ & \\
\hline Disponibilidade & $\begin{array}{l}\text { O OA deve ser indexado e armazenado de } \\
\text { maneira que possa ser facilmente encontrado. }\end{array}$ & $\begin{array}{l}\text { LORI, } \\
\text { CEdMA }\end{array}$ \\
\hline Acessibilidade & $\begin{array}{l}\text { O OA pode ser acessado por diferentes } \\
\text { dispositivos, diferentes contextos (ex.: velocidade } \\
\text { de conexão diferente) e, principalmente, possuir } \\
\text { versão adaptada para diferentes tipos de } \\
\text { usuários (deficientes visuais, idosos etc.). }\end{array}$ & LORI, \\
CEd & $\begin{array}{l}\text { O OA deve apresentar resultados precisos, } \\
\text { dentro do esperado. }\end{array}$ & $\begin{array}{l}\text { ISO/IEC } \\
9126\end{array}$ \\
\hline Precisão & $\begin{array}{l}\text { O OA não deve possuir falhas técnicas. } \\
\text { Confiabilidade }\end{array}$ & $\begin{array}{l}\text { ISO/IEC } \\
9126\end{array}$ \\
\hline $\begin{array}{l}\text { Facilidade de } \\
\text { instalação } \\
\text { (installability) }\end{array}$ & \begin{tabular}{l} 
O OA deve ser fácil de ser instalado. \\
\hline O OA deve funcionar em diversos cenários \\
como: diferentes sistemas operacionais, \\
diferentes ambientes virtuais de Avaliação, \\
diferentes hardware etc.
\end{tabular} & $\begin{array}{l}\text { ISO/IEC } \\
9126\end{array}$ \\
\hline Interoperabilidade & $\begin{array}{l}\text { O OA deve poder interagir com outros OA ou } \\
\text { sistemas. }\end{array}$ & $\begin{array}{l}\text { ISO/IEC } \\
9126\end{array}$ \\
\hline Usabilidade & $\begin{array}{l}\text { O OA deve ser fácil de ser utilizado e estar de } \\
\text { acordo com os padrões mais consagrados de } \\
\text { usabilidade. }\end{array}$ & $\begin{array}{l}\text { ISO/IEC } \\
912\end{array}$ \\
\hline
\end{tabular}

Como modelo, o presente estudo acorda com Kuhn (2006, p. 43), no qual assume que "o seu uso estabelecido, um paradigma é um modelo ou padrão aceito". Logo,modelos se situam no cerne das ciências, como $\mathrm{De} \operatorname{Re}(2000)$ advoga, em que o pensamento descritivo é representado pelos modelos físicos, enquanto o pensamento argumentativo é representado pelos modelos matemáticos. Assim, modelos são criados, aceitos ou rejeitados, podendo suplantar os existentes, mas independente da sua atuação, são representações científicas.

\section{DESENVOLVIMENTO DE OBJETOS DE APRENDIZAGEM}

O desenvolvimento de OAs requer atenção pedagógica que transcende as questões tecnológicas da web. $O$ projeto e 
implementação de conteúdos e atividades instrucionais devem seguir princípios cognitivos, de modo que os aprendizes tenham melhor assimilação e retenção (SWELLER; Van MERRIEMBOER; PAAS:1998). Assim, conteúdos, apresentação e atividades devem estar em consonância com o público alvo para ter um melhor aproveitamento.

O planejamento do OA visa garantir a consonância do OA com seu público alvo e envolve a definição das características dos aprendizes, os elementos disponíveis no ambiente virtual de aprendizagem e as características dos instrutores, como sua disponibilidade e nível de apoio e conhecimento. Assim como o escopo do conteúdo a ser abordado, o tipo de sequência em que os conteúdos serão apresentados aos aprendizes são aspectos que devem ser englobados no planejamento, como ilustrado na figura 1.

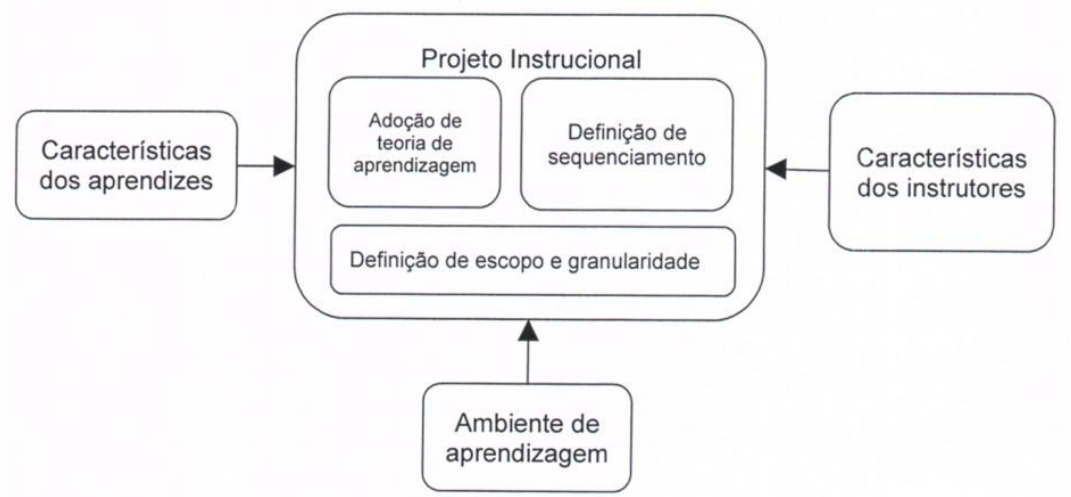

FIGURA 1 - Elementos para o planejamento de um objeto de aprendizagem FONTE: os autores

As escolhas sobre o público-alvo, o ambiente e o apoio dos instrutores definirão o tipo de OA a ser desenvolvido, que pode se enquadrar em diferentes tipos: do tipo simples, que não permite modificações e pode ser usado em diferentes contextos; do tipo combinado intacto, que agrega até quatro objetos simples e apresenta possibilidade de reuso em diferentes contextos; do tipo combinado modificável, que agrega objetos de aprendizagem e inclui estratégias motivacionais e de interação entre OAs, com pouca possibilidade de aplicação em outros contextos; do tipo apresentação adaptativa, que incorpora lógica e arquivos de dados que orientam a apresentação dos conteúdos; e do tipo instrução 
adaptativa, que engloba características dos objetos de apresentação adaptativa, mas com simulações para avaliação (WILEY, 2000).

Assim, a capacidade de um sistema, nesse caso o OA, de ser utilizado com eficácia, constitui no ponto importante do seu desenvolvimento. Itens de usabilidade afetam diretamente os resultados de aprendizagem, devendo ser respeitados elementos que atendam a diferentes estilos de aprendizagem (SANTOS e SCHNEIDER, 2012). Ressalta-se que um OA digital é constituído por artefatos de software, com os quais o aprendiz interage, e esses devem ser construídos de forma a potencializar o aprendizado.

Para obter tais resultados, o desenvolvimento de OAs para web pode ocorrer por meio do projeto de conteúdo instrucional, em que a etapa final é implementada por meio da aplicação de técnicas como a codificação em HTML, scripts e folhas de estilo do tipo CascadingStyleSheets (CSS). Assim, os elementos instrucionais concebidos e desenvolvidos podem ser incorporados e arranjados de acordo com um projeto gráfico e de interação, utilizando objetos multimídias, textuais e outros, como ilustrado na figura 2.

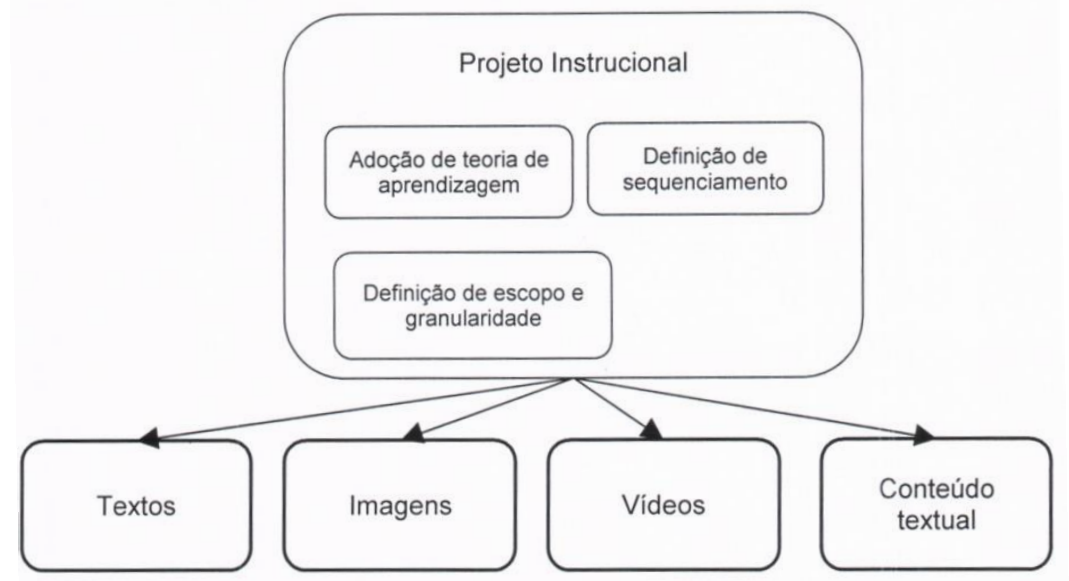

FIGURA 2 - Incorporação de elementos multimídia em OA em função do projeto instrucional

Ao encadear o projeto instrucional e a implementação na web, o desenvolvimento de OAs requer uma equipe multidisciplinar, de forma a obter maior eficácia e atender aos critérios de qualidade levantados. Como relata Filho e Filho (2014), o desenvolvimento de 
OA para web requer projeto equilibrado entre conteúdo e forma de apresentação, com usabilidade adequada aos aprendizes, os quais revelam a complexidade ligadas aos aspectos cognitivos, envolvendo questões como: a informação a ser divulgada; a tarefa a ser realizada; o tempo necessário para as atividades; os espaços possíveis para as interações e o conteúdo elencado de maneira significativa.

Durante o projeto e implementação, diferentes perfis profissionais, tais como o especialista no conteúdo, o designer instrucional, o projetista gráfico, o webdesigner e programadores atuam colaborativamente sobre um objeto final que é o material para aprendizagem. A dinâmica de desenvolvimento pode ocorrer de forma iterativa, de modo que o conteudista e o designer instrucional tenham maior liberdade para experimentar alternativas de organização e apresentação dos conteúdos.

Nessas experimentações, pequenas mudanças no conteúdo, como a adição de uma página intermediária, podem demandar a modificação de todas as páginas posteriores. A mudança de um elemento gráfico, como um ícone presente em todas as páginas, requer a modificação em todos os itens já produzidos. Com isso, ressalta-se, além da necessidade de planejamento no desenvolvimento de OA, a aplicação de um modelo de desenvolvimento que facilite a implementação do OA a partir dos materiais com o conteúdo abordado em um cenário de desenvolvimento iterativo.

Da mesma forma, a incorporação de material multimídia, como imagens ou vídeos, exige conhecimentos de codificação que não são competência do conteudista ou do designer instrucional. Ao mesmo tempo, realizar esta incorporação em tempo de desenvolvimento e sem depender diretamente de programadores permite ao designer instrucional e ao conteudista um refinamento do conteúdo e de suas formas de apresentação.

A próxima seção descreve o modelo e as técnicas que visam facilitar a realização de modificações para experimentações iterativas, tanto no desenvolvimento de conteúdos, quanto na estrutura e na apresentação de materiais instrucionais.

\section{MODELO PARA DESENVOLVIMENTO DE OAS}

Conceitualmente, o ciclo previsto para o desenvolvimento do OA, após seu projeto instrucional, incorpora repetidas etapas de 
prototipagem, com experimentação de diferentes alternativas na camada de apresentação; modificações na granularidade dos elementos do OA, com a distribuição do conteúdo em novas páginas ou novos módulos; a inclusão de elementos multimídia, incorporando-se ou modificando imagens, vídeos, animações, simulações e outros;a mudança em elementos gráficos, como a alteração de um ícone de navegação que está presente em todas as páginas; modificações no sequenciamento do conteúdo, alterandose a ordem de apresentação dos tópicos de conteúdo; e testes e análises heurísticas, que permitem validar ou coletar subsídios para aprimoramentos do OA em novos ciclos de desenvolvimento até a obtenção de resultados satisfatórios aos usuários, sob o ponto de vista da ergonomia da interface ou de usabilidade dos conteúdos. Este ciclo, representado na figura 3, pode ser repetido de forma ágil e quantas vezes forem necessárias até se obter um OA adequado ao projeto instrucional.

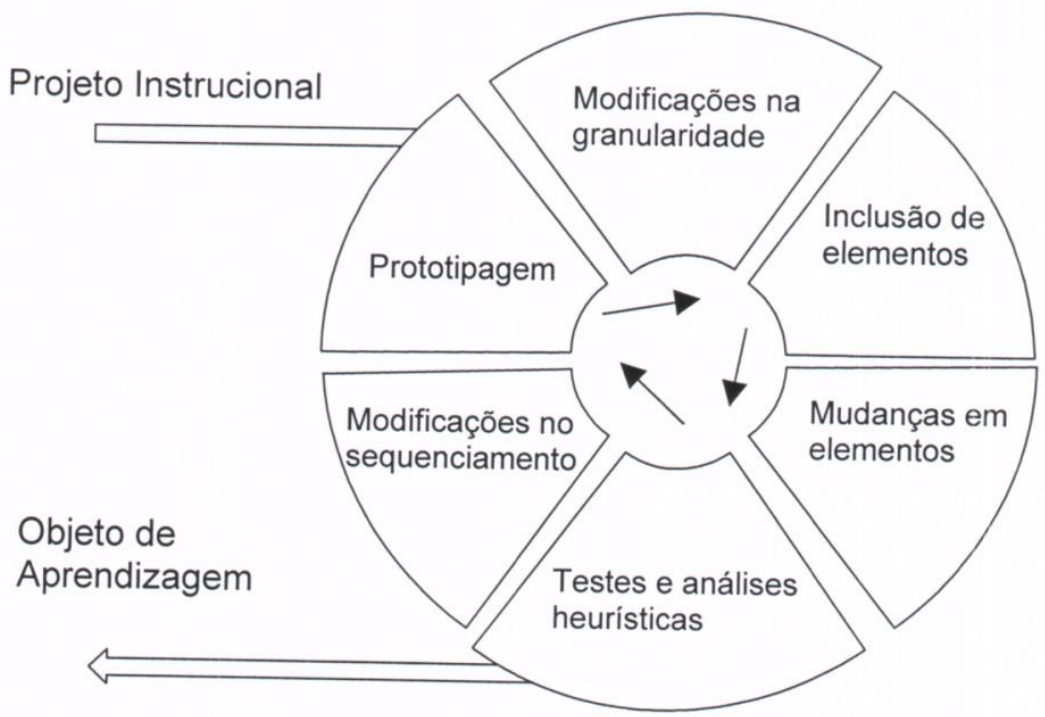

FIGURA 3 - Modelo de ciclo iterativo no desenvolvimento do AO FONTE: os autores

Para facilitar a transição entre os diferentes ciclos de iteração no desenvolvimento de um objeto de aprendizagem, é proposto um método baseado no uso de:

- Templates gráficos, que descrevem a aparência e 
arquitetura de informação do OA;

- Texto estruturado com rótulos personalizados, que agrupam o conteúdo do OA em blocos de conteúdo e definem seus elementos multimédia;

- Parser, processo que aplica o texto estruturado no template gráfico, incorporando os elementos multimédia.

Os tópicos seguintes descrevem cada um destes elementos e como se relacionam no desenvolvimento de um OA.

\section{O TEMPLATE GRÁFICO}

O template gráfico consiste num esqueleto básico a ser preenchido com os conteúdos e elementos de navegação do OA, apresentando os locais em que os elementos derivados da arquitetura da informação que orienta a navegação do OA devem ser posicionados, assim como de áreas destinadas aos textos, imagens e elementos multimédia. $O$ template define também comportamentos como pop-ups de mensagens e de exibição de imagens e links auxiliares. Um exemplo de template básico é apresentado no diagrama da figura 4:

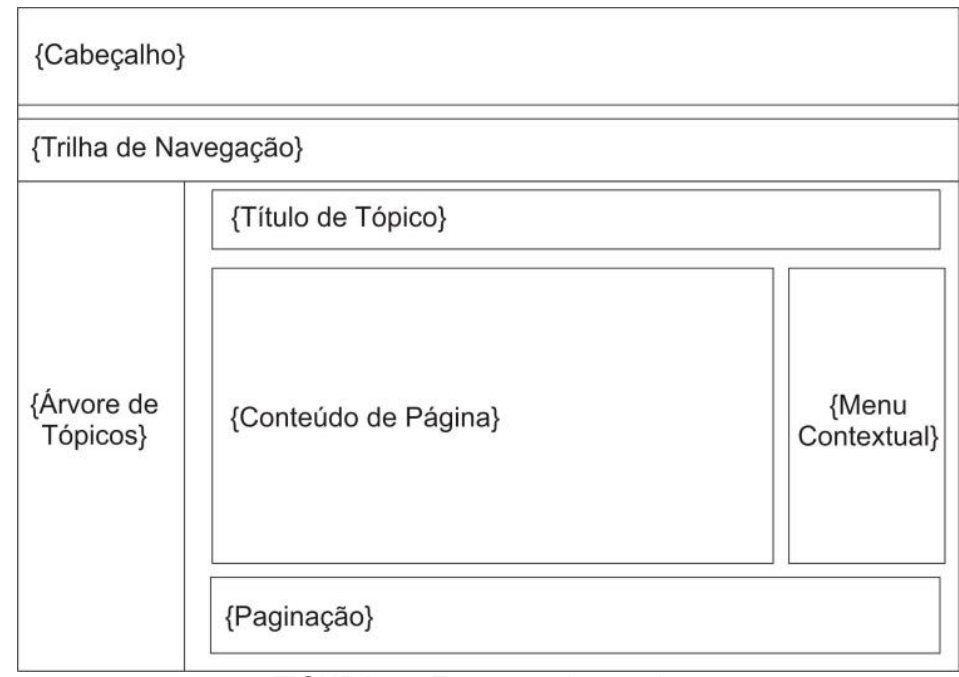

FIGURA 4 - Esquema de template

No caso do template apresentado é possível identificar áreas destinadas a diferentes tipos de conteúdos e elementos de navegação. O topo das páginas estará destinado a apresentar uma imagem fixa, logo abaixo, uma trilha de navegação exibirá o 
contexto de navegação onde o usuário se encontra. Uma árvore de tópicos fica disponível no lado esquerdo da tela, que, em seu centro, apresenta o conteúdo da página, apoiado por um menu contextual, que pode, por exemplo, apresentar links para documentos auxiliares ou outros recursos de apoio à aprendizagem.

O template apresentado é um exemplo, sendo que novos templates podem ser desenvolvidos de acordo com as demandas modeladas para outros objetos de aprendizagem. Cada curso pode ter seu próprio template, adequado aos conteúdos a serem apresentados. Ou também OA pode ser entregue em diferentes formatos, podendo haver um template para versão em PDF, versão em formato e-PUB ou mesmo no padrão SCORM.

O próximo tópico descreve o texto estruturado com rótulos personalizados, que irá representar os conteúdos e sua estrutura que serão apresentados pelo OA.

\section{Texto Estruturado com Rótulos Personalizados}

O processo inicial na implementação do conteúdo do OA, segundo o modelo proposto, baseia-se em um texto, no qual os objetivos pedagógicos, público alvo e conteúdos são definidos. Esse texto é então enriquecido com uma linguagem de marcação com rótulos personalizados. Como casos similares, apresentamos algumas aplicações que fazem o uso de linguagens de marcação, como o Latex, voltado à elaboração e à diagramação de artigos científicos, e ilustrado na plataforma colaborativa ShareLateX da figura 5 . 


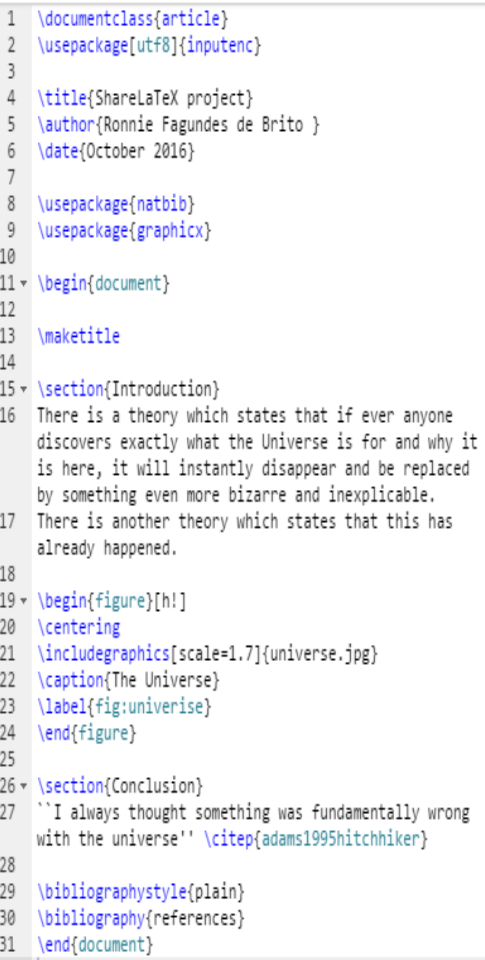

FIGURA 5 - Editor Latex Colaborativo.

FONTE: https://pt.sharelatex.com/

Outro software que faz uso de linguagem de marcação é o Dillinger, voltado ao desenvolvimento de documentação técnica e apresentado na figura 6 . 


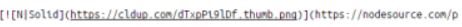

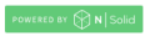

Dillinger is a cloud-enabled, nobile-ready, offline-storage, Angular Js

powered HTMLS Markdown editor.

Dillinger is a cloud-enabled, mobile-ready, offline-storage, AngularJS powered HTML.5

Markdown editor.

Type sone Narkdown an the left

See HTMC in the right

Nogic

You can also:

- Invort and save files from Cittub, Dropbox, Coogle ortive and one orive

Drog and drap files tinto otllinger

- Export docunents as Markdown, HTNe and PDF

Markdown is a lightweight narkup languape based on the fornatting conventions

that people naturally use in enatl. As [John Gruber] writes on the [Markdown

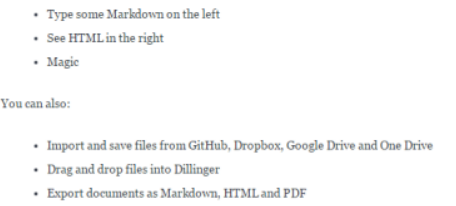

FIGURA 6 - Editor de linguagem de marcação Dillinger

FONTE: http://dillinger.io/

Esses softwares permitem gerar documentos em diferentes formatos, por exemplo, o Dillinger, que possibilita exportar 0 documento em formato HTML ou PDF, e fazem uso de linguagem de marcação com comandos próprios.

No modelo proposto, a estruturação do texto deverá indicar os elementos que serão aplicados no template gráfico e a forma com que estes elementos serão aplicados. É no texto estruturado que se definem os módulos, tópicos e páginas a serem encapsulados no OA.

Um exemplo de texto estruturado é apresentado na figura 7. Cada tela inicia com o rótulo <tela $>$, que dá título ao tema apresentado e delimita o conteúdo. Rótulos como <figura >, <video> e <figuratexto> agregam os elementos multimídias a comporem 0 objeto educacional.

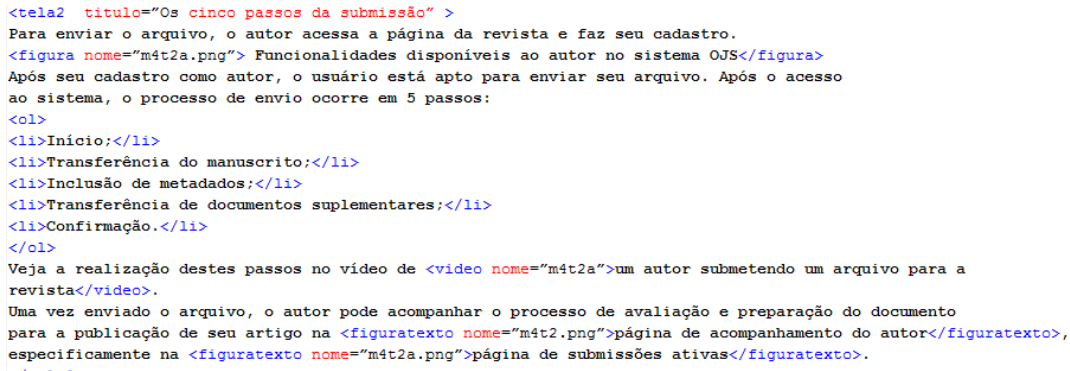

FIGURA 7 - Detalhe de um texto estruturado com tags 
Novos rótulos da linguagem de marcação podem ser desenvolvidos em cada cenário, por isso denominados de rótulos personalizados. Como exemplos, podemos enumerar alguns rótulos propostos para conteúdo multimédia, cada um com um objetivo específico descrito no quadro 3.

QUADRO 3 - Rótulos personalizados propostos para 0 desenvolvimento de material de OA

\begin{tabular}{|c|c|c|}
\hline Objetivo & Rótulo & Exemplo \\
\hline Inserir uma imagem & <imagem> & $\begin{array}{l}\text { <imagem arquivo="arquivo.png"> } \\
\text { Legenda da imagem } \\
</ \text { imagem }>\end{array}$ \\
\hline Inserir um vídeo & $<$ video $>$ & $\begin{array}{l}\text { <video nome="arquivo"> } \\
\text { Avaliação } \\
\text { </video> }\end{array}$ \\
\hline $\begin{array}{ll}\text { Definir } & \text { uma } \\
\text { atividade } & \end{array}$ & <atividade> & $\begin{array}{l}\text { <atividade> } \\
\text { Enumere os exemplos de rótulos } \\
\text { aplicáveis } \\
\text { </atividade> }\end{array}$ \\
\hline $\begin{array}{l}\text { Incluir a definição } \\
\text { contextual de um } \\
\text { palavra }\end{array}$ & <saibamais> & $\begin{array}{l}\text { A <saibamais } \\
\text { id='foto'>Fotossíntese</saibamais> é } \\
\text { realizada pelas plantas. } \\
\\
\text { <saibamais id='foto'> } \\
\text { Síntese de moléculas orgânicas a partir } \\
\text { do dióxido de carbono atmosférico e da } \\
\text { água, utilizando a luz como fonte de } \\
\text { energia. } \\
</ \text { saibamais }>\end{array}$ \\
\hline
\end{tabular}

Além dos rótulos personalizados, rótulos tradicionais utilizados em HTML5 podem ser usados para formatar o texto, com rótulos de parágrafos, listas, ou outros.

A principal vantagem no uso dos rótulos personalizados é a simplificação da incorporação de materiais multimédia. Por exemplo, ao adicionar um elemento de vídeo no conteúdo, o autor utiliza a tag '<video>' acompanhada de alguns atributos. Ou, então, para inserir um link a uma imagem que aparece em popup, o autor utiliza a tag<figuratexto>, também acompanhada de alguns atributos. Esses rótulos são convertidos em código HTML 5, como apresentado no quadro 4 abaixo, de forma que o desenvolvedor do conteúdo pode abstrair os detalhes específicos de programação para a 
incorporação de tais elementos em uma página a ser apresentada na web.

QUADRO 4 - Exemplos de rótulos utilizados no material do OA para o curso sobre OJS

\begin{tabular}{|c|c|}
\hline Rótulo & Código \\
\hline $\begin{array}{l}<\text { video } \\
\text { nome="ojs_avaliador">Avali } \\
\text { ação</video> }\end{array}$ & $\begin{array}{l}\text { <a class="videoopener" } \\
\text { title="<imgsrc='videolcon.png' />" } \\
\text { id="ojs_avaliador">avaliador acessando o } \\
\text { OCS e avaliando um trabalho</a> } \\
\text { <video id="ojs_avaliador" width="640" } \\
\text { height="480" controls="true" } \\
\text { poster="ojs_avaliador.jpg" preload="'> } \\
\text { <sourcetype="video/webm" } \\
\text { src="ojs_avaliador.webm"> } \\
\text { <sourcetype="video/mp4" } \\
\text { src="ojs_avaliador.mp4"> } \\
\quad \text { <sourcetype="video/ogg" } \\
\text { src="ojs_avaliador.ogv"> <imgalt="o } \\
\text { Editor de Texto aceitando as modificações } \\
\text { e notificando a conclusão da edição de } \\
\text { texto" src="ojs_avaliador.jpg" } \\
\text { style="position:absolute;left:0;" width="640" } \\
\text { height="480" title="'> < </video> }\end{array}$ \\
\hline $\begin{array}{l}<\text { figuratexto } \\
\text { nome="savants.jpg">Journ } \\
\text { aldesSavants</figuratexto> }\end{array}$ & $\begin{array}{l}<\text { span class="yoxview"><a } \\
\text { onclick="_gaq.push(['_trackEvent', 'Figura', } \\
\text { ',',',','View','Journal des Savants' ]);" } \\
\text { class="yoxviewLink" title=""' } \\
\text { href="savants.jpg">Journal des } \\
\text { Savants }</ \text { a }></ \text { span }>\end{array}$ \\
\hline
\end{tabular}

Os rótulos personalizados permitem uma rápida modificação do conteúdo, em formato textual complementado por marcadores, os quais indicam o posicionamento dos elementos multimédia a serem incorporados. O conjunto de rótulos básicos pode ser definido para cada cenário, devendo haver o mapeamento entre o esquema de aplicação do rótulo no texto e o código HTML5 resultante.

O processo de conversão entre o rótulo e o código é realizado pelo processo que denominamos "parser", que é descrito na próxima seção. 


\section{O PARSER}

No processo de "parsing", é tradicionalmente associado à análise sintática de um texto de determinada linguagem, o qual deve seguir suas regras formais. Na informática, o parsing está inserido no processo de compilação de um programa de computador, transformando seu código-fonte em um código executável.

No modelo proposto, o parsing se aplica na transformação do texto estruturado, validando os rótulos utilizados e transformando-os em códigos HTML 5.

Desta forma, o parser adiciona os elementos multimídias que enriquecem o conteúdo, além de fornecer a forma de apresentação. Ao final desse processo, tem-se um material instrucional, que comporá o objeto de aprendizagem. O processo é apresentado na figura 8 .

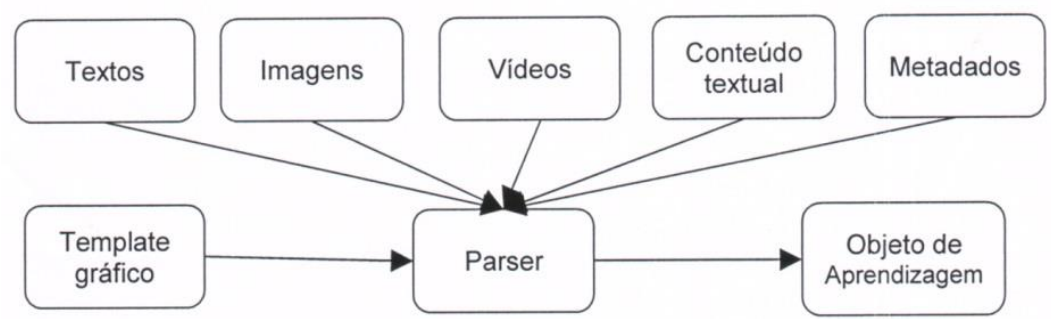

FIGURA 8 - Fluxo de parsing que incorpora material multimedia nos objetos de aprendizagem

O processo de parse não é intensivo em recursos computacionais e pode ser realizado indefinidas vezes, permitindo a experimentação de diferentes etapas do material que comporá o OA, e para diferentes templates gráficos, podendo gerar diferentes formatos de apresentação a partir do mesmo texto estruturado.

Com esse modelo de desenvolvimento de OA, é possível operar em colaboração, já que cada profissional pode atuar distintamente em uma parte do fluxo. Cada peça é criada com base em um documento de texto, no qual o conteúdo é exposto. Assim, cada profissional pode atuar, sugerindo a criação de elementos que são agregados, compondo ao final um OA totalmente portável, podendo ser transformados em pacotes, seguindo o padrão SCORM ou outro associado.

O processo deve ser acompanhado por toda a equipe, de forma a obter o melhor resultado, visto tratar-se de um processo 
colaborativo, mesmo que cada profissional tenha maior envolvimento em um elemento ou etapa. A produção de um objeto educacional, como peça a compor um objeto de aprendizagem, deve ser colaborativo e multidisciplinar para garantir atendimento a critérios de qualidade estabelecidos.

Após o processo de parse, tem-se a apresentação como na figura 9, com a integração entre o texto e elementos multimídia devidamente formatados de acordo com o template gráfico.

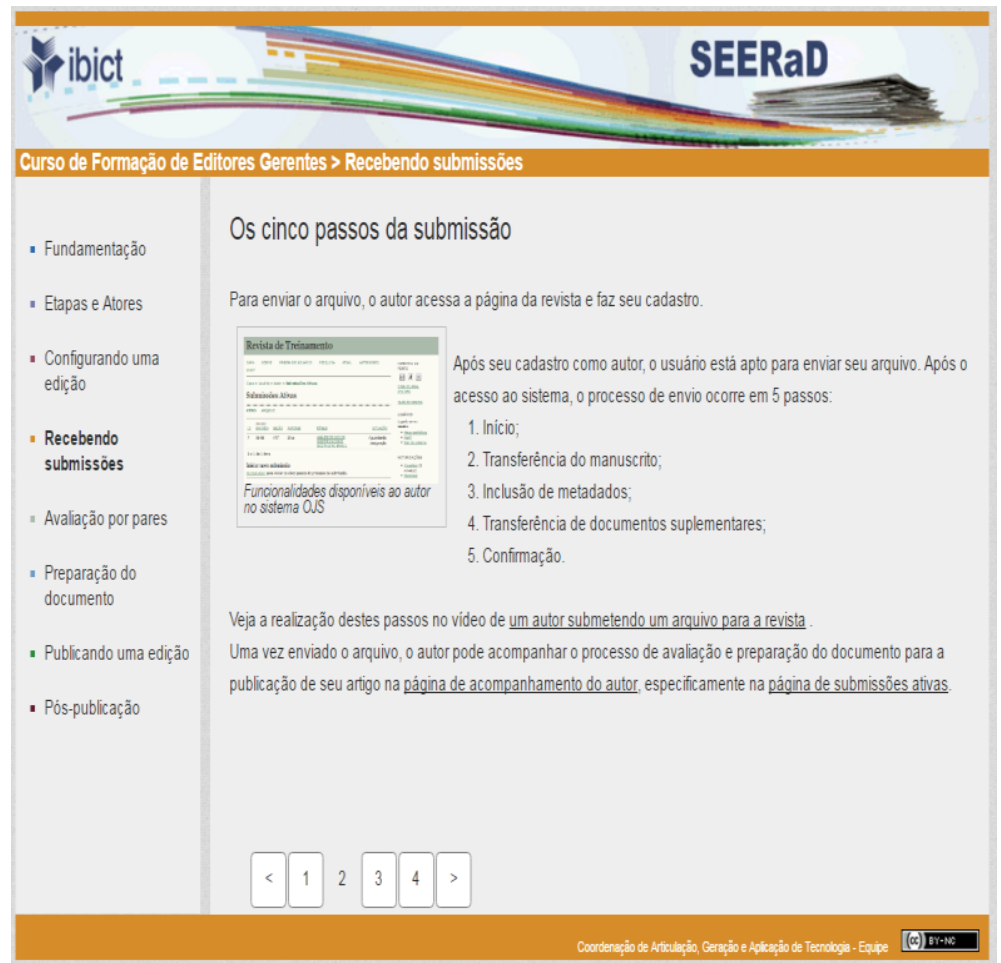

FIGURA 9 - Resultado do processamento do texto estruturado

Modificações no conteúdo da tela, como a incorporação de um novo vídeo ou figura, ou mesmo a adição de novas telas intermediárias, podem ser realizadas pela edição do texto e realização de novo parsing, que gerarão novas páginas HTML, de acordo com rótulos aplicados no texto. 


\section{IMPLEMENTAÇÃO DO CURSO SOBRE OPEN JOURNAL SYSTEMS}

O Open Journal Systems é um sistema de apoio à editoração de revistas em meio digital e amplamente adotado na comunidade acadêmica brasileira, onde também é conhecido como SEER (Sistema Eletrônico de Editoração de Revistas). O IBICT oferece formação para o uso deste sistema e, como meio de atender a esse objetivo, é oferecido um curso à distância sobre OJS.

Para a elaboração do material de apoio a esse curso, que está disponível em http://ead.ibict.br/cursos/seer2/, foi aplicada a metodologia aqui apresentada.

O template gráfico utilizado é ilustrado na figura 10. Nesse template, foi definido que cada módulo do curso teria uma cor associada. Essa cor é atribuída a cada tele durante o parsing e registrada em uma folha de estilos.

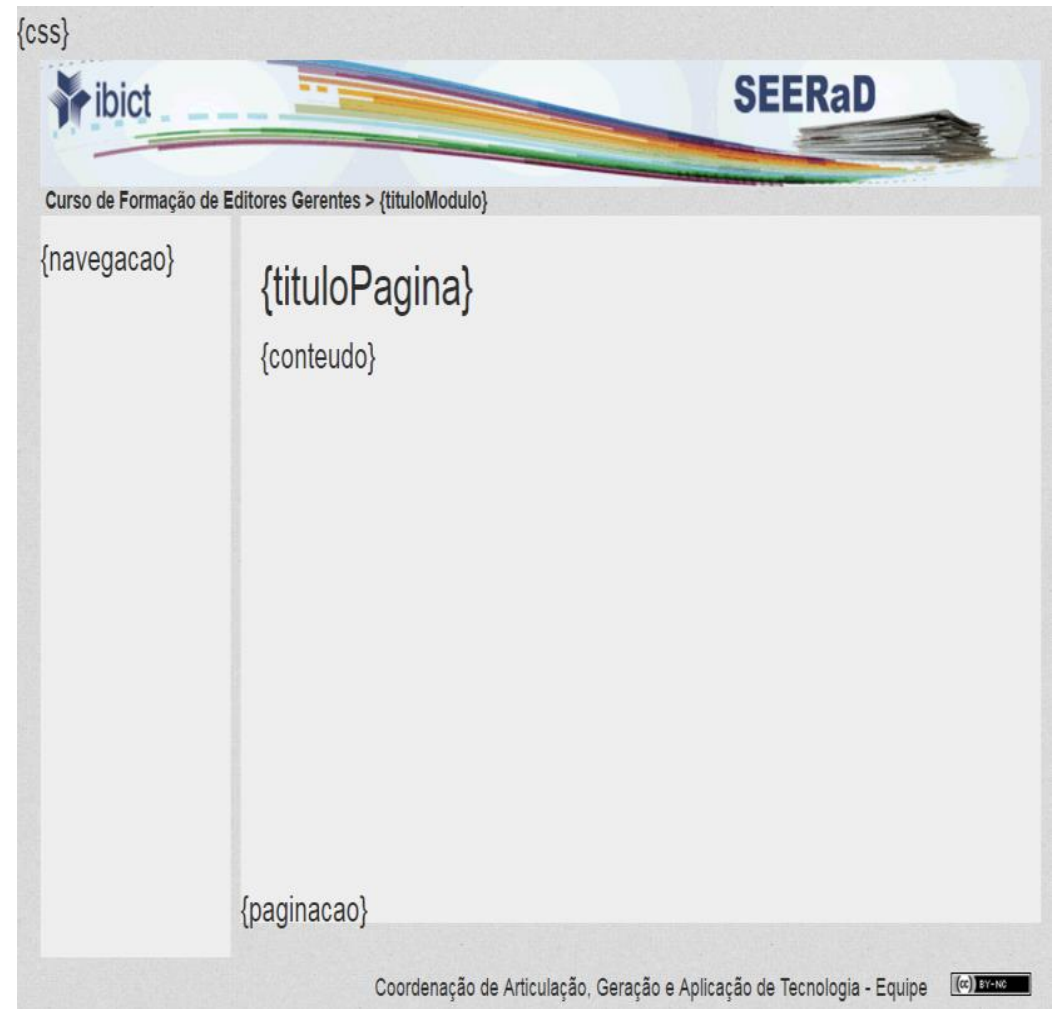

FIGURA 10 - Template do curso SEERaD 
Além do template gráfico, foi criado um conjunto de rótulos personalizados, enumerados no quadro 5 abaixo.

QUADRO 5 - Rótulos para os elementos utilizados na estruturação do material instrucional

\begin{tabular}{|l|l|}
\hline Rótulo & Objetivo \\
\hline$<$ curso $>$ & Define o conteúdo global do curso \\
\hline$<$ modulo $>$ & $\begin{array}{l}\text { Defino as unidades do curso. Cada Módulo } \\
\text { consiste de várias telas. }\end{array}$ \\
\hline$<$ tela $>$ & $\begin{array}{l}\text { Corresponde a uma tela apresentada ao usuário. } \\
\text { O total de telas do módulo orienta sua paginação. }\end{array}$ \\
\hline$<$ biblio $>$ & $\begin{array}{l}\text { Adiciona link contextual para visualização de } \\
\text { referência bibliográfica. }\end{array}$ \\
\hline$<$ figuratexto $>$ & $\begin{array}{l}\text { Cria um link no texto, que ao ser acionado exibe } \\
\text { uma imagem. }\end{array}$ \\
\hline$<$ figura $>$ & $\begin{array}{l}\text { Cria uma imagem apresentada ao lado do } \\
\text { conteúdo textual e com o texto encapsulado como } \\
\text { legenda. }\end{array}$ \\
\hline$<$ citacao $>$ & $\begin{array}{l}\text { Coloca o trecho de texto englobado pelo rótulo } \\
\text { com formatação diferenciada. }\end{array}$ \\
\hline$<$ video $>$ & $\begin{array}{l}\text { Converte o trecho de texto em um link que ao ser } \\
\text { acionado exibe uma camada com um vídeo, } \\
\text { inicialmente pausado. }\end{array}$ \\
\hline$<$ link $>$ & $\begin{array}{l}\text { Adiciona um link a uma URL externa ou a outra } \\
\text { página do conteúdo. }\end{array}$ \\
\hline$<$ dica $>$ & $\begin{array}{l}\text { Adiciona um link no trecho de texto encapsulado } \\
\text { que ao ser acionado apresenta uma camada }\end{array}$ \\
\hline$<$ atividade $>$ & $\begin{array}{l}\text { Adiciona formatação gráfica de enunciado de } \\
\text { atividade no texto encapsulado. }\end{array}$ \\
\hline & \\
\hline &
\end{tabular}

Como exemplo de texto estruturado é apresentado um trecho de conteúdo, na figura 11, no qual uma página possui um vídeo e uma imagem. Há também um marcador de "saibamais", que é um elemento hipertextual que exibe uma mensagem quando 0 usuário clica sobre o link correspondente. 


\section{- 〈tela3 titulo="Criando a Revista" $\rangle$}

A primeira etapa para implementar seu periódico é requisitar

ao administrador do portal SERR/OJS 〈video nome $=" m 2 t 3 a ">a$ criação de sua revista $\langle$ video〉.

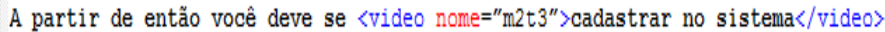

por meio do 〈dicaCadastro〉formulário de cadastro</dicaCadastro〉 e pedir ao administrador do portal a sua $\left\langle v i d e o\right.$ nome $\left.=" m 2 t 3 c^{\prime \prime}\right\rangle$ designação como Editor Gerente $\langle/$ video〉

Caso perca sua senha, você pode usar o recurso de recuperação de dados de acesso, no link Acesso> Esqueceu a senha.

Ao informar o e-mail que usou no seu cadastrado o sistema irá gerar uma nova senha e enviá-la para seu endereço eletrônico.

\section{- $-\langle$ dicaCadastro〉}

0 formulário de cadastro de usuário é usado por todos os tipos de papéis do sistema, caso não sejam cadastrados pelo administrador.

No formulário há a possibilidade de escolha entre Autor, Avaliador ou Leitor. 〈figura nome="m2t3a.png">Tela com formulário de cadastro do usuario 〈/figura〉 Estas opções permitem no caso de:

$21 \boxminus\langle u l \times l i>$ Leitor: ser notificado via e-mail da publicação de nova edição da revista; $</ 1 i\rangle$

22 〈li〉Autor: submeter artigos à revista; $\langle/ 1 i\rangle$

23 - $\langle$ li〉Avaliador: realizar avaliação pelos pares das submissões a esta revista. $\langle/ 1 i\rangle /$ ul $\rangle$

24 No caso de cadastro como avaliador, recomendado informar as áreas de interesse para avalia $25-\langle/$ dicaCadastro $\rangle$

$26\langle/$ tela3 $>$

FIGURA 11 - Trecho de texto estruturado do material para o curso sobre OJS

Conforme definido no modelo, o processo de parsing gerará as telas do material do curso a partir do texto estruturado. Exemplos dessas telas são apresentados nas imagens seguintes, cuja figura 12 apresenta a tela gerada a partir do texto estruturado apresentado na figura 11. 


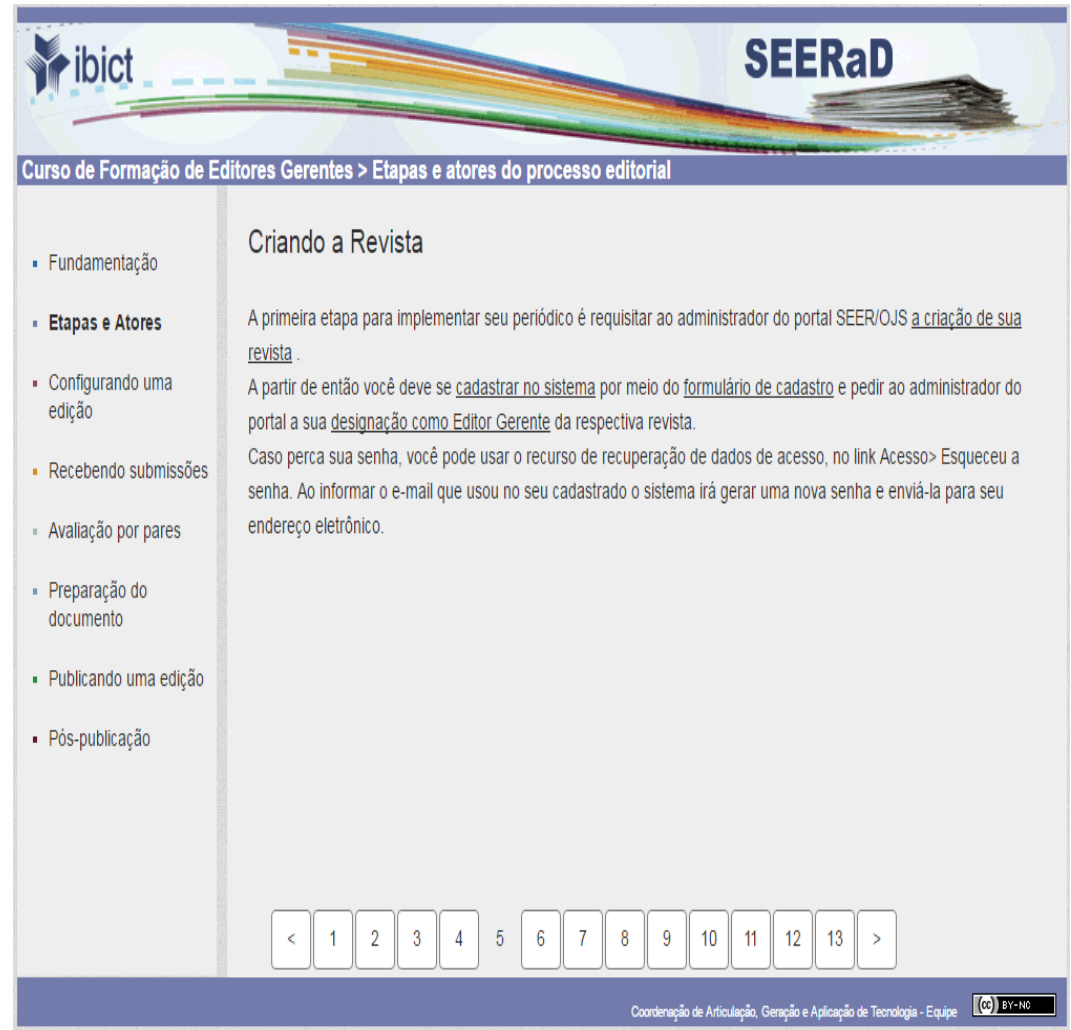

FIGURA 11 - Tela do material do curso sobre OJS/SEER gerada a partir de texto estruturado

Elementos de vídeo são apresentados como uma camada sobre a tela principal, com opção de legenda e fechamento da tela. A figura 13 apresenta o vídeo definido na linha 5, do código da figura 11. Os vídeos são acompanhados de uma legenda, no formato SRT (SubripSubtitleFormat), que é exibida concorrentemente aos vídeos que demonstram as funcionalidades do sistema, visto que esses não possuem áudio. 


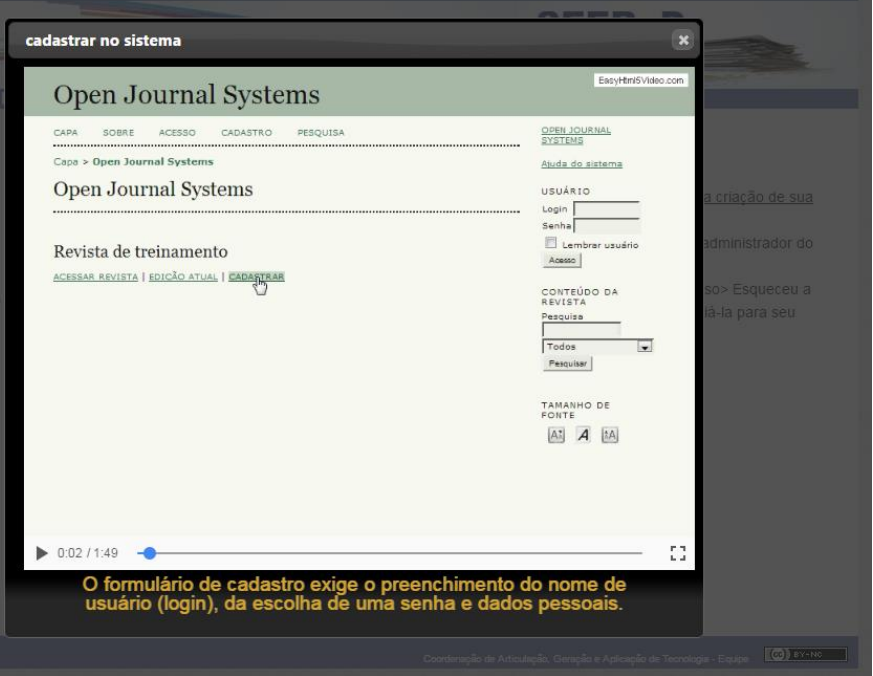

FIGURA 13 - Exibição de elemento vídeo associado a texto do material do curso sobre OJS

Além do vídeo, o curso utiliza o rótulo < dica>, que adiciona uma explicação adicional no texto. Um exemplo de apresentação de dica é vista na figura 14.

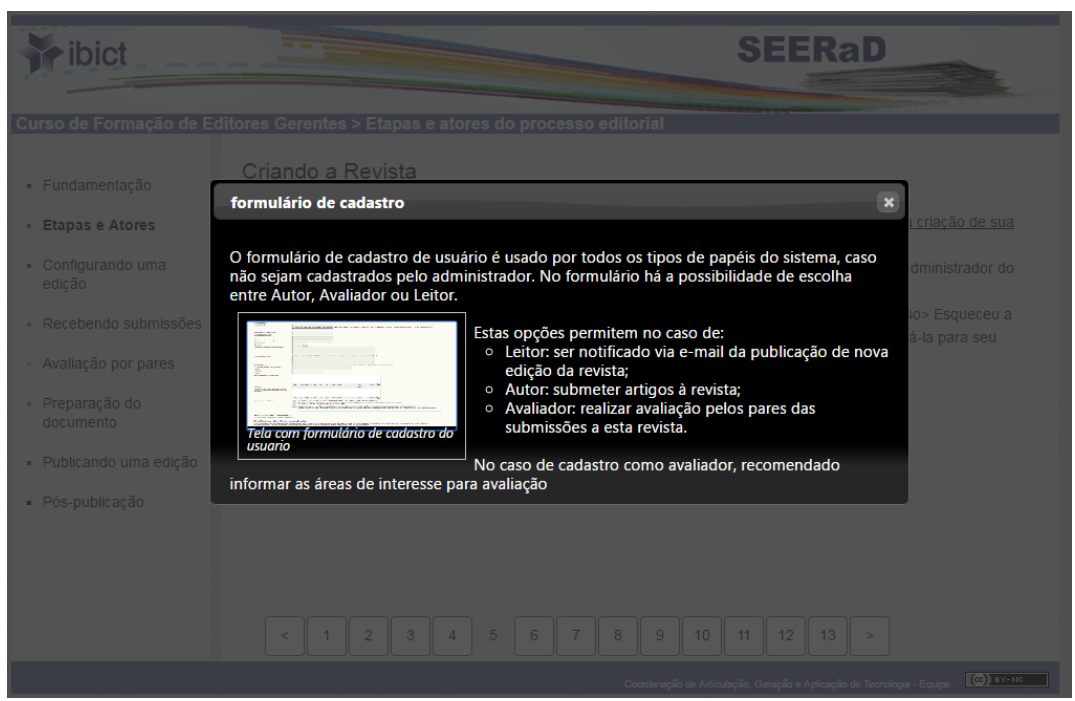

FIGURA 14 - Exibição de elemento de explicação adicional associado a texto do material do curso sobre OJS. 
O material gerado a partir desse método foi utilizado no curso para editores gerentes para o OJS em seis edições. Os cursistas foram estimulados a responder a um questionário de avaliação de material hipermídia, baseado em Elissavet e Economides (2003), alinhados aos critérios elencados por Braga et al (2012), em que obtivemos alguns resultados positivos quanto ao material do curso, apresentados a seguir.

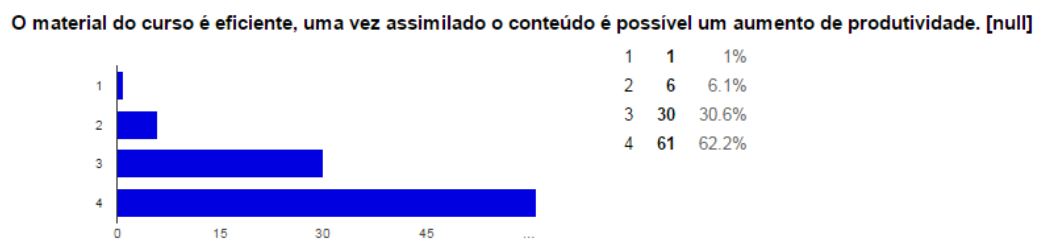

A qualidade do texto, imagens, gráficos e videos permite boa legibilidade e visualização. [null]

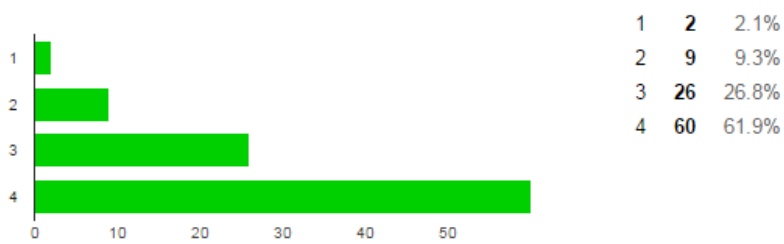

O material é segmentado de forma que apresenta questöes, revisöes e resumos de cada segmento. [null]

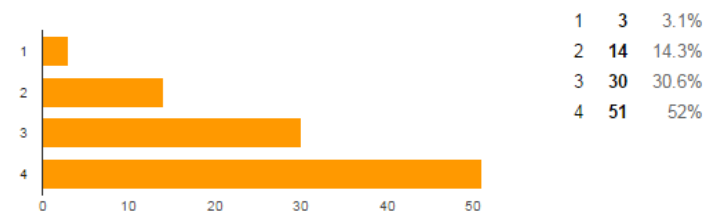

A estrutura do conteúdo permite navegar livremente entre diferentes topicos, pemitindo avançar, ver exemplos e revisitar os materiais apresentados. [null]

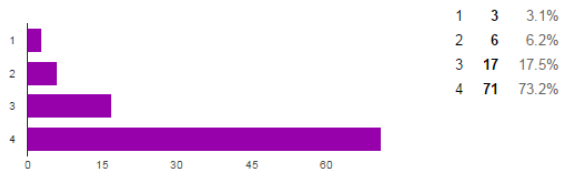

\section{CONCLUSÕES E PERSPECTIVAS FUTURAS}

O processo de elaboração de conteúdos, planejamento de atividades e a implementação de materiais instrucionais, utilizando os recursos oferecidos pela web, exige conhecimentos nem sempre 
dominadas por todos os membros das equipes envolvidas.

De forma a facilitar questões relacionadas a linguagens e aspectos técnicos, apresentamos um método para desenvolvimento de material instrucional que visa otimizar a etapa de implementação de conteúdo multimídia para a plataforma web.

O método baseia-se no uso de tags personalizadas e simplificadas na elaboração dos conteúdos, as quais são posteriormente traduzidas em códigos compatíveis com a web.

Assim, permitindo maior liberdade de experimentação por parte dos desenvolvedores de conteúdo, prescindindo a necessidade de conhecimento sobre recursos de programação, como Javascript, folhas de estilo e tags HTML para incorporação de vídeo e outros elementos multimídia, os quais são atendidos pelo uso de tags personalizadas e de templates gráficos.

O método é aplicado no desenvolvimento de material instrucional para treinamento de editores gerentes de revistas que utilizam o Open Journal Systems, e já foi utilizado em 6 edições do curso.

Além de oferecer maior facilidade na elaboração de materiais instrucionais, o método apresentado permite gerenciar de forma mais eficiente alterações e reformulações nos materiais. A criação de novas versões, correções de erros e aprimoramentos é também facilitada com o método aplicado. Outra vantagem do método proposto é a possibilidade de geração do conteúdo em diferentes formatos, como PDF e HTML, bastando modificar 0 template utilizado.

Como limitação, identificamos que o domínio do vocabulário introduzido pelos rótulos personalizados é uma atividade que, mesmo de forma simplificada, demanda preparo por parte dos desenvolvedores.

A complementação dos rótulos personalizados por meio do uso de elementos nativos do HTML, no meio do texto estruturado, implica, em um primeiro contato, poluição visual, que interfere na leitura do texto estruturado. Entretanto, acreditamos que as facilidades oferecidas por esse método se sobrepõem aos desafios apresentados para sua utilização.

\section{REFERÊNCIAS}

ANDRADE, Fabiano Viana; DE ALMEIDA LOPES, Arilise Moraes. EaD: uma história de inovações tecnológicas no Brasil. In: Congresso de Tecnologia da Informação. 2013. 
BRAGA, J. C. et al. Desafios para o Desenvolvimento de Objetos de Aprendizagem Reutilizáveis e de Qualidade. In: Anais do Workshop de Desafios da Computação Aplicada à Educação. 2012. p. 90-99.

Elissavet, G., \&Economides, A. A. (2003).An Evaluation Instrument for Hypermedia Courseware. Educational Technology \&Society, 6(2), 31-44, (ISSN 1436-4522)

FILHO, Vilson Martins; FIALHO, Francisco Antonio Pereira. Design de experiência educacional. Novas abordagens no design de objetos educacionais.

Pereira, E.W., e Moraes, R.A. (2009). História da educação a distância e os desafios na formação de professores no Brasil. In: Souza, A. M., Fiorentini, L. M. R. y Rodrigues, M. A. M. (Org.).. Educação superior à distância: comunidade de trabalho e aprendizagem em rede, 3, pp. 65-90. Brasília: Universidade de Brasília, Faculdade de Educação.

Santos, G. A., \& Schneider, H. N. (2012). E-learning: Recommendations for instructional designers and interface considering learning style. Paper presented at the Proceedings of the 6th Euro American Conference on Telematics and Information Systems, EATIS 2012

SARAIVA, Terezinha. Educação a distância no Brasil: lições da história. aberto, Brasília, ano, v. 16, p. 17-27, 1996

Silveira, I.F., Araújo Jr, C. F., Amaral, L. H., Oliveira, I. C. A. (2003) Development of Reusable Learning Objects. In: Proceedings of ITHET - 4th International Conference on Information Technology Based Higher Education and Training, pp. 301-305. Marrakech, Marrocos.

Sweller, J.; van Merrienboer,J. J. G.; Paas, F.G. W. C.. Cognitive Architecture and Instructional Design.EducationalPsychologyReview. September 1998, Volume 10, Issue3, pp 251-296

TESTA, M.G. (2002). Fatores críticos de sucesso de programas de educação a distância via internet. Dissertação de Mestrado, Universidade Federal do Rio Grande do Sul, Porto Alegre, RS, Brasil.

Wiley, David.2000 https://opencontent.org/docs/dissertation.pdf 
